\title{
Arborescences
}

Revue d'études françaises

\section{Gérald Leblanc et l'expérience du corps dans la ville}

\section{Andrée Mélissa Ferron}

Numéro 3, juillet 2013

Lire le texte et son espace : outils, méthodes, études

URI : https://id.erudit.org/iderudit/1017369ar

DOI : https://doi.org/10.7202/1017369ar

Aller au sommaire du numéro

Éditeur(s)

Département d'études françaises, Université de Toronto

ISSN

1925-5357 (numérique)

Découvrir la revue

Citer cet article

Ferron, A. M. (2013). Gérald Leblanc et l'expérience du corps dans la ville. Arborescences, (3). https://doi.org/10.7202/1017369ar

\section{Résumé de l'article}

Dans La condition urbaine, Olivier Mongin soutenait que « le premier langage qui permet de qualifier l'expérience urbaine est [...] celui du poète et de l'écrivain, celui des mots et de leur rythmique. » (Mongin 2005 : 32). Mongin posait alors la question de l'implication du corps dans l'expérience de l'espace urbain traduite dans le texte. En littérature acadienne, il est difficile de songer à un exemple plus pertinent pour illustrer ces propos que celui de l'oeuvre de Gérald Leblanc. Dans un contexte de francophonie minoritaire, où l'espace demeure investi d'un récit de dépossession, Leblanc aura choisi de canaliser l'identité collective dans les possibles de l'expérience urbaine individuelle. Empirique, adoptant l'esprit jubilatoire que lui inspire l'espace monctonien pour libérer son identité acadienne de ses faux récits contraignants et restrictifs, le poète propose une « infinité de parcours » individuels (Mongin 2005 : 39-40) pour signifier l'appartenance collective. 


\title{
GÉRALD LEBLANC ET L'EXPÉRIENCE DU CORPS DANS LA VILLE
}

\author{
Andrée Mélissa Ferron \\ University of Alberta
}

Dès son institutionnalisation en 1972, la littérature acadienne s'est revendiqué une importante identité urbaine. À la toute première œuvre publiée par une maison d'édition acadienne, alors que Raymond Guy Leblanc faisait paraitre l'emblématique Cri de terre, la ville s'est d'emblée imposée comme sujet. Déjà, elle étouffait l'écriture, laissant pressentir un sentiment d'envahissement dont le poète alors ne pouvait s'affranchir. Dans la littérature acadienne, la ville de Moncton prime dans l'expression de l'imaginaire urbain. C'est d'ailleurs ce qu'affirme Marie-Linda Lord :

Aborder l'identité et l'urbanité dans la littérature acadienne, c'est avant tout explorer Moncton dans la littérature acadienne. Le récit urbain dans la littérature acadienne est un récit monctonien qui est fondé à la fois sur l'observation et l'imaginaire. [...] Le noyau de tension qu'est Moncton avec son urbanité est l'un des quatre sociogrammes majeurs de la littérature acadienne qui sont investis par plusieurs écrivains, à la fois sur le plan discursif et le plan idéologique. Les trois autres sont le pays/le territoire, la langue/le chiac, et l'américanité/le continent. (Lord $2006: 67$ )

Il s'agit donc de déterminer comment la ville/Moncton, inspirant le corps et l'esprit, ${ }^{1}$ en vient à influencer considérablement les configurations de chacune des trois autres thématiques récurrentes. ${ }^{2}$ Cette réflexion propose une analyse exhaustive en ce sens. Toutefois, nous nous en tiendrons aux réflexions et aux observations qui ont alimenté notre problématique de départ, notamment en ce qui a trait à l'expérience du corps dans la ville, et les principes de sa transposition dans le texte littéraire acadien.

Dans La condition urbaine, Olivier Mongin note que la ville des écrivains n'est pas celle, par exemple, des ingénieurs-urbanistes. Chez les écrivains, l'expérience urbaine engage le corps (Mongin 2005 : 26-27) et implique la pratique du lieu (selon de Certeau). Ces deux façons d'appréhender la ville donne lieu à deux appellations différentes, amenant Mongin à distinguer la «ville-sujet» des écrivains à la «ville-objet» de ceux qui la pensent sans l'expérimenter (Mongin 2005 : 26). Dans son « envoi » à la première partie de La Condition urbaine, il explique :

\footnotetext{
${ }^{1}$ Nous paraphrasons : « ces villes idéalisées qui inspirent encore nos corps et nos esprits » (Mongin $2005: 18$ ).

2 Terme que nous choisissons dans le cadre de cet article.
} 
La ville entendue comme expérience urbaine est polyphonique. C'est d'abord une expérience physique, la déambulation du corps dans un espace où prime le rapport circulaire entre un centre et une périphérie. L'expérience urbaine, c'est ensuite un espace public où des corps s'exposent et où peut s'inventer une vie politique par le biais de la délibération, des libertés, et de la revendication égalitaire. [...] Or cette expérience multidimensionnelle ne sépare pas le public et le privé, mais les associe. C'est cette expérience, entendue selon ces divers registres, que nous évoquons ici en tant que première appréhension de la condition urbaine. (Mongin $2005: 21-22$ )

Mongin insiste sur le fait que l'expérience urbaine «entrelace l'individuel et le collectif», et qu'elle «met en rapport, dans une sorte de dialectique "inachevable”, des éléments opposés : l’intérieur et l'extérieur, le dedans et le dehors, le centre et la périphérie, le privé et le public ». (Mongin 2005 : 29) Cet entrelacement est d'autant plus inéluctablement envisageable dans le contexte acadien, car comme le souligne François Paré, on ne remet jamais en question «le rapport d’intimité usuel entre la collectivité et l'écrivain» (Paré 2001 [1993] : 134) au sein d’une littérature qui se définit comme exiguë, ou issue d'un contexte minoritaire.

De fait, l'expérience urbaine première selon Mongin, serait celle du «corps individuel qui sort de lui-même pour s'aventurer dans un corps collectif [...] : l'histoire d['un] corps qui se sent "relié" à une ville, l'histoire des corps qui créent un espace commun » (Mongin 2005 : 30). Ainsi, la ville serait définie selon une pluralité de subjectivités ou de consciences individuelles :

Si le corps est l'image qui surgit spontanément quand le poète ou le phénoménologue évoquent la ville, si le cœur de la ville bat à un rythme plus ou moins soutenu, s'il peut connaître l'arythmie ou un battement excessif, il y a autant de poétiques de la ville que de corps qui la parcourent et s'y aventurent. Et dans tous les cas, l'écriture corporelle parcourt des villes qui se présentent elles-mêmes comme des livres. (Mongin 2005 : 37-38)

Mongin propose ainsi de définir l'expérience urbaine comme une «infinité de parcours» (Mongin 2005 : 39-40) à l'intérieur d'un espace circonscrit. Or, dit-il, selon la subjectivité du corps déambulant, ces parcours sont ponctués ou guidés par certains lieux personnels distinctifs qui dénotent une « "image mentale” qui se forme progressivement et se confond avec l’idée même de la ville. » (Mongin 2005 : 42-43) Ces lieux se rattachent ainsi à l'histoire de la ville, à son rythme identitaire. En nous basant sur les propos de Mongin, nous pourrions affirmer qu'en offrant la possibilité d'une nouvelle cartographie marquée par la présence et l'appartenance, la ville serait un moyen de s'affranchir du statut de minoritaire pour tenter d'établir celui d'égalitaire ${ }^{3}$ :

La ville existe quand des individus parviennent à créer des liens provisoires dans un espace singulier et se considèrent comme des citadins. Si la ville a un nom propre qui l'identifie et la

\footnotetext{
${ }^{3}$ Non seulement sur les plans linguistique ou culturel mais aussi sur le plan de l'identité sexuelle en ce qui a trait à l'œuvre de Leblanc.
} 
singularise, elle est en même temps plurielle, traversée par des rythmes différenciés. (Mongin 2005 : 48-49)

Comme le note Robert Major dans la préface à la troisième édition de Les littératures de l'exiguïté de François Paré, le sentiment de déracinement ou de non-appartenance « dessille les yeux, rend conscient et engage à la réflexion» (Paré 2001 [1993] : 11). Or, en Acadie, la prise de parole suivant la prise de conscience est passée par le territoire, et Paré ne manque pas de souligner plus loin dans son ouvrage, que « les petites littératures tendent à glorifier l'espace » (Paré 2001 [1993] : 115) parce que «l'espace est prégnant de sens » (Paré 2001 [1993] : 116). Dans le cas de la littérature acadienne, cet énoncé est aisément vérifiable pour la simple raison qu’on le retrouve dans le contexte de l'héritage d'une déportation, donc d'une dépossession de l'espace. Les écrivains de l'exiguïté, souligne Paré, (et il nomme Gérald Leblanc et Guy Arsenault entre autres) «font de la dépossession collective une condition de richesse pour l'écriture poétique » (Paré 2001 [1993] : 139). En Acadie littéraire - et de façon proéminente en poésie acadienne, la prise en charge de la thématique du territoire dans le contexte urbain implique une certaine forme d'autonomisation de la conscience de soi, énoncée sur le plan de la redéfinition identitaire. Autrement dit, il était question de revoir les nouvelles réalités de l'acadianité:

de dire à nouveau nous vivons ici

que la lueur des rues illumine nos histoires

(Leblanc, Éloge du chiac: 117)

Benoit Doyon-Gosselin notait sur l'arrivée du roman postmoderne acadien : «[...] à partir des années 1980, les romanciers mettent à profit des espaces autres pour échapper au cadre restreint qu'offrait la fiction acadienne» (Doyon-Gosselin 2007 : 371). En fait, il s'agissait de marquer, pour reprendre les mots de François Paré, « le triomphe de l’imaginaire sur le réel » (Paré 1998 : 19). Paré rappelle le portrait qu'avait tracé Alain Masson de l'Acadie de l'avenir, dans les années 1980 :

L'Acadie ne sera pas un État, instance purement administrative qui n'intéresse ni la littérature, ni le «peuple acadien » dans son agir quotidien. Elle ne sera pas non plus un avatar folklorique. Plutôt, $[\ldots]$ la communauté acadienne prendra les contours d'une urbanité civile et civilisée, loin de l'enfermement national [...] Ainsi se formulait alors le projet singulier de toute une génération de poètes, qui, au «mythe » enfermant d'une Acadie villageoise et taciturne, celui que véhiculait encore largement le discours culturel acadien, qui voudrait substituer la «légende » d'une collectivité « arrivée en ville», qui ferait un «tintamarre », et se donnerait ainsi bruyamment à lire. (Paré 1998 : 21)

De plus, « les petites littératures semblent jeter tout leur dévolu sur l'écrivain, dont l'œuvre et les prestations publiques sont au cœur des représentations de l'agir collectif » (Paré 2001 [1993] : 182). Ceci nous ramène vers notre problématique de départ, à savoir le corps individuel qui se prolonge dans le corps collectif en situation minoritaire, et ce, au sein de l'espace urbain représenté par Moncton. 
Nous amorcerons notre analyse en évoquant brièvement l'une des premières œuvres ayant témoigné de l'expérience physique et linguistique de la ville de Moncton, à savoir celle de Guy Arsenault, et du recueil Acadie Rock. CEuvre pionnière par son contenu, sa forme, ses mots et son esthétique, sa parution en 1973 eut l'effet d'une onde de choc sur la très 'jeune' littérature acadienne de l'époque. La poésie d'Arsenault présentait une écriture habitée de subjectivité et de sensations issues d'un espace qui tardait à faire son apparition dans le texte littéraire acadien, à savoir la backyard urbaine d'Acadie. La backyard se traduisait en fait en l'espace individualisé, l'intime et le privé mis en rapport avec le public. Jusqu'alors, l'espace et le territoire en littérature acadienne avaient été des sujets de collectivité. Avant Acadie Rock, il y avait eu Cri de terre de Raymond Guy Leblanc, où la ville était toujours l'espace de l'Autre dans lequel ni le je, ni le nous n'avait encore réussi à se placer. Or, c'est à partir de Guy Arsenault que l'espace éclate, se fragmente, et tend à devenir sujet d'une individualité. Et si l'on se réfère aux grandes thématiques majeures de la littérature acadienne, il nous semble tout à fait possible d'affirmer qu'au moment où paraît Acadie Rock débute la conceptualisation de Moncton comme sujet à part entière, et non une simple composante du thème du pays/territoire. Dans l'œuvre où s'affirmait pour la première fois l'expérience assumée de la « condition urbaine » de l'Acadie, il importait de s'en tenir à certaines étapes essentielles : se situer, nommer l'espace, s'y rattacher au moyen d'une démarche empirique, empirisme primaire mais non pas naï.

Comme bon nombre d'écrivains qui ont publié leurs œuvres à la suite d'Acadie Rock, Gérald Leblanc se revendiquait de l'œuvre de Guy Arsenault. Il a su reconnaittre, lors d'un entretien avec Michel Giroux en 1992, que la publication d'Acadie Rock a été l'un des événements les plus importants de la littérature acadienne (Giroux 1992 : 148). Se confiant à Giroux, Leblanc a voulu expliquer la genèse de son projet poétique, dont les préoccupations selon lui étaient communes à plusieurs jeunes poètes de l'époque :

Lorsque j'ai écrit mes premières œuvres, j'avais la conviction qu'on était en train de construire quelque chose. Je dis « on », c'est à dire « nous »: Herménégilde Chiasson, Guy Arsenault, Raymond Leblanc, France Daigle, Diane (sic) [Léger], Régis Brun, et j'en passe. Nous avions le projet de faire une littérature acadienne. On s'inscrivait dans un courant qui faisait rupture en parlant de l'Acadie telle qu'elle était ressentie au 20e siècle pour bon nombre d'Acadiens. Il n'était plus question, pour nous, de cages à homards au bout du quai. C'était plutôt vivre en ville, c'était l'expérience des drogues, etc., un travail de défrichage, même si à l'époque on ne pensait pas à ce que nous faisions en ces termes. Je me disais que mon travail était un fragment d'un plus grand morceau. (Giroux 1992 : 161)

Chez Gérald Leblanc, l'esprit combatif s'est rapidement dissout pour laisser place à un empirisme optimiste; il n'était plus question de lutter contre son espace mais plutôt d'en faire l'expérience au quotidien, de l'habiter en le rendant « habitable ». Il n'était plus question non plus de lutter contre la 
mouvance de l'époque vers «l'américain »; Leblanc ayant d'ailleurs toujours revendiqué une identité américaine pour l'Acadie. ${ }^{4}$ L'écriture de Leblanc décourageait le confinement - sur tous les plans et proposait une relation harmonieuse avec le continent, ce qui donnait lieu à une meilleure compréhension de l'espace en soi. L'esprit du premier recueil de Leblanc, Comme un otage du quotidien, est en quelque sorte résumé dans les vers :

s'accumulent des perceptions la mémoire en strates chavirées les temps passés et présents se dénouent et s'embrassent gestation des images dans les multiples réalités d'un corps

(Leblanc, Comme un otage du quotidien: 35 )

Ces vers peuvent être perçus comme l'annonce d'une Acadie nouvelle déconstruite et reconstruite dans un projet d'écriture actualisant. Les écrivains acadiens qui ont émergé des années soixante-dix et qui allaient devenir les pionniers d'une nouvelle littérature acadienne ont paradoxalement eu la chance de trouver refuge dans une crise de la forme et des genres, ce qui leur a permis de façonner à leur guise la spécificité de la littérature acadienne, tout en s'assurant d'une reconnaissance institutionnelle en Acadie et ailleurs dans la francophonie mondiale. ${ }^{5}$ En commentant la poésie de son époque, Claude Beausoleil écrivait « des mots anglais traversent parfois le décor. On est comme dans la vie. » dans son texte d'ouverture des actes du colloque Les cent lignes de notre américanité tenu à Moncton du 14 au 16 juin 1984 (Beausoleil 1984 : 8-9). Une écriture «comme dans la vie » sera le souci le plus grand du travail poétique de Gérald Leblanc, parce qu'elle transmet avec le plus d'intensité le message fondamental d'une littérature émergente et d'un peuple marginal ; celui, en effet, d'être. Dans Paysages imaginaires d'Acadie, Raoul Boudreau et Mylène White présentent l'œuvre de Leblanc en ces mots :

Les références aux lieux géographiques, aux espaces réels, sont l'un des moyens privilégiés par lesquels s'effectue cet ancrage dans l'univers concret. On ne sera donc pas étonné de relever dans cette poésie une prolifération des noms de lieux et d'espaces divers qui lui donne un encadrement précis et rigoureux alors que ce genre littéraire nous avait plutôt habitué aux délimitations floues du discours intérieur. Cependant, l'œuvre de Leblanc comporte ce para-

\footnotetext{
${ }^{4}$ Voir, par exemple, le poème « carte » à la page 13 du recueil Complaintes du continent.

${ }^{5}$ Raoul Boudreau résume le phénomène avec beaucoup de lucidité : «Les poètes acadiens des années soixante-dix ne pouvaient s'appuyer sur une tradition littéraire véritablement constituée et ils traînaient le lourd héritage d'une langue stigmatisée, quasi-honteuse et d'un sentiment d'extraterritorialité collé à la peau. Cette poésie a quand même pu prendre son envol en raison, pour une bonne part, de la conjoncture favorable créée par la montée du postmodernisme et la perte de confiance dans les grands récits, notamment dans le discours nommé Littérature et la valorisation corollaire des discours marginaux, minoritaires et hétérogènes. Émergeant au cœur d’une vaste crise de langage, la poésie acadienne trouvait spontanément à faire servir sa propre expérience d'un langage inadéquat. » (Boudreau 1998 : 12).
} 
doxe que les lieux y sont aussi la source et l'objet d'une rêverie, d'une expansion de l'imaginaire, bref d'une recréation artistique qui est au cœur de l'écriture littéraire. Si dans l'œuvre de Leblanc l'écrivain s'apparente au cartographe et qu'il a pour mission de baliser le territoire, de construire l'espace, ce phénomène n'est pas étranger à l'expérience de l'exiguité en général, et à l'expérience acadienne de la dépossession du territoire en particulier. (Boudreau et White 2009 : 43)

Si le projet avait été commencé par Guy Arsenault, il prendra réellement forme chez Gérald Leblanc. Dans son œuvre, Moncton devient définitivement - au sens où l'entend Mongin - ville-sujet au sein du texte littéraire acadien. Alimentée par la déambulation dans la ville, l'intimité d'un appartement, la musique, les drogues et le fait d'être Acadien à Moncton, l'écriture colle à l'événement, et met en place un univers imaginaire très personnalisé ; le langage y est livré à tous les fantasmes tout en étant largement imprégné de littéralité. La plupart des éléments que l'on retrouve au sein de la poésie de Leblanc sont également présents dans son seul roman, Moncton Mantra: le même genre de «texteintervention» (Bolduc 1985: 53), cette écriture de l'aujourd'hui, le sensible et l'incongru traduits dans la mise en forme des mots: le lyrisme étendu sur un canevas de musique. Chez Leblanc, l'expérience urbaine est très individualisée. Le collectif s'y manifeste de manière plus subtile, différente de ce que l'on a pu lire dans Acadie Rock. Bien que le poète procède la plupart du temps de manière tout aussi empirique, s'emplissant des sons, des odeurs, des sensations, et des images de la ville pour la marquer de sa présence, il y a chez Leblanc, cette composante intérieure à laquelle font allusion Boudreau et Whyte : la ville rêvée, imaginée, fantasmée. Moncton y est à la fois réelle et irréelle; elle prend des proportions souvent démesurées, ou encore elle n'a tout simplement plus de fin, comme l'exprime le premier vers de « musique d'attente » du recueil Éloge du chiac:
ville de moebius
où chaque parole nous revient en poème
chaque image en vidéo
sur l'écran cathodique de nos projets
nous cartographions jusqu'à l'étourdissement
notre passé rythmé au présent
dans l'attente suspendue des musiques
à la veille de commencer
(Leblanc, Éloge du chiac: 94)

Ou encore, dans le poème «multipiste » de l'Extrême frontière, où Moncton est le lieu de possibilités infinies, à la mesure d'un désir infini :

Moncton serait cet espace

où j'ai traversé le rouge dans toutes ses dimensions

réseaux dévorants des accidents de parcours

rages et rumeurs de l'émotion

lyrisme du trop-plein

ville de mes vies parallèles 
Moncton multipiste

dans l'immense Amérique de mon désir

(Leblanc, l'Extrême frontière : 106)

L'œuvre de Gérald Leblanc s'impose d'elle-même lorsque l'on transpose dans le contexte acadien les propos d'Olivier Mongin, qui, nous le répétons, soulignent qu'« il y a autant de poétiques de la ville que de corps qui la parcourent et s’y aventurent. » (Mongin 2005 : 37). Chez Leblanc, le collectif trouve sa place non plus dans un soulèvement de groupe mais dans le sentiment de liberté qui se trouve associé à l'espace urbain de Moncton. Les poèmes proposent un parcours dans l'espace qui se révèle essentiellement individuel, mais cette individualité est elle-même une invitation destinée au collectif. Dans l'espace monctonien de Leblanc, l'Acadie est libérée, à la fois des lourdeurs du passé et des contraintes identitaires. La ville devient l'ouverture sur le continent. ${ }^{6}$ Chez Leblanc, l’Amérique est un désir et Moncton en est le condensé.

Dans le rêve et le fantasme, l'espace se redéfinit selon une forme désirée, libérée, il est sujet au désir et sujet à la rythmique particulière du poète. L'œuvre de Leblanc cristallise la liberté associée à la condition urbaine. Si la backyard d'Arsenault à l'époque prenait le sens d'une présence collective qu'on individualisait et qu'il fallait assigner à l'espace (« comme si tou'l monde se connaissait »), ${ }^{7}$ chez Leblanc, elle est marquée d'une pluralité de vies et de sens, d’individualités multiples :

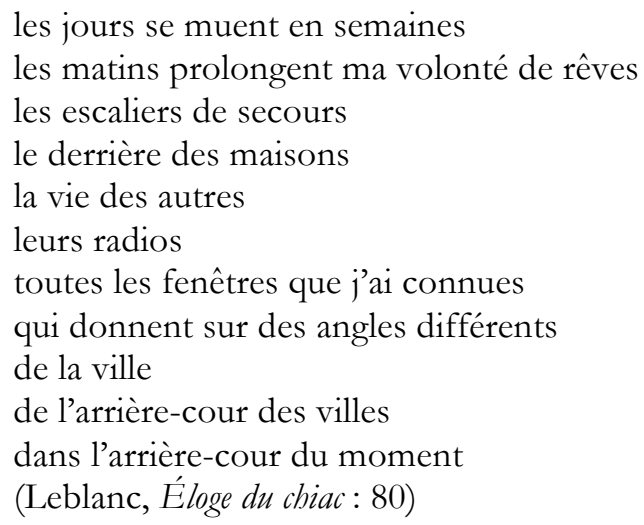

On remarque que le mot n'est plus «backyard», - alors qu'il aurait pu l'être - mais arrière-cour. Leblanc fait usage du chiac lorsqu'il le veut bien, et refuse d'obéir aux contraintes linguistiques, identitaires ou autres. Le fait que le recueil porte le titre Éloge du chiac ne change rien à la liberté d'écriture

${ }^{6}$ Comme le notait René Plantier : «Lorsque Ponge invente des rapports neufs avec les objets cachés sous des mots usés, il joue sur des cohésions de signifiants, il révèle des associations dans le code d'une langue ; lorsqu'un Acadien joue dans l'américanité, c'est le lyrisme personnel qui unifie ou tente d'unifier dans les connotations, les tensions des langues et des cultures. » (Plantier $1988: 137)$.

7 Tiré du poème «Tableau de backyard » (Arsenault $1973: 31$ ). 
que le poète se donne. ${ }^{8}$ L'éloge, en fait, devait être énoncé, dans la forme d'un titre, d'un poème, sans toutefois devenir une contrainte de l'écriture, qui se doit d'être libre même si elle est acadienne. Le poète souhaite donc simplement faire exister l'esprit de tolérance et de liberté que lui inspire son espace urbain. ${ }^{9}$ D’où les propos de Boudreau et de White qui parlaient d'espace rêvé et fantasmé — le rêve et le fantasme se rattachent aux possibilités de l'espace, et moins aux conditions réelles qui s'y retrouvent. Selon les textes de Leblanc, si le chiac est parlé à Moncton, son apparition dans les bouches et les textes acadiens ne devrait pas faire l'objet d'un soulèvement d'indignation. Leblanc, répétons-le, rejette les contraintes et les identités préfabriquées :

de jouer dans la langue et d'en rire

d'en rêver quand on find out

qu'on communique

même si le voisin fait mine

de ne rien comprendre

too bad de se priver

de pareille façon

de faire accroire

contre soi-même

que ce rythme n'existe pas

la musique est o.k.

le monde itou

on dirait que toute

est à la bonne place

c'est slick so

stick around

(Leblanc, Éloge du cbiac : 11)

Pour le poète, il n'est plus question de prescrire une identité acadienne selon des repères trop bien définis. Dans le Moncton de son Amérique, l'Acadie, l'Acadien, peuvent essayer d'exister librement, sans trop penser comment. Du moins ce souhait reste formulé, mais plus ou moins réalisable :

Ce que j'aimerais leur dire, c'est que chez nous, il est impossible d'oublier que l'on est Acadien. [...] Je n'ai pas le goût de me lamenter devant eux, quoique, à l'intérieur de moi-même, je me dis que j’aimerais ne plus penser à ça pour un an, pour deux ans, pour toujours... (Leblanc, Moncton Mantra: 61-62)

Plus loin dans Moncton Mantra, on lit :

\footnotetext{
${ }^{8}$ Clint Bruce précisera : «En préconisant un "éloge du chiac", Leblanc récuse d'entrée de jeu deux fondamentalismes : celui des loyalistes anglophones, défenseurs d'un Canada anglais uniculturel, ainsi que le purisme bien-pensant de l'élite acadienne. L'appel à la tolérance à l'égard de la langue passe tout d'abord par une liberté absolue dans la langue [...]. Étant donné qu'il se parle dans une ville marquée par des tensions ethnolinguistiques particulières et en est même le résultat, le chiac s'impose comme emblème et incarnation même d'une tolérance qui peut bien sûr évoluer en échange. Ce vernaculaire, issu du contact entre de l'anglais et du français acadien n'est-il pas, de par sa nature même, une production transculturelle? »(Bruce 2005 : 211).

${ }^{9}$ Voir à ce sujet l'article de Boudreau (2007).
} 
De plus en plus, je décèle un courant qui nous ramène vers cette glorification du passé. Je veux bien connaittre notre passé; je trouve néanmoins ce retour en arrière inquiétant. Comme si nous pouvions nous défaire de la vie moderne pour reprendre la vie des bois, comme le proclament certains. Comme si chaque soirée, il devenait obligatoire de sortir le violon, de jouer des cuillères et de chanter des chansons à répondre. L'épine dorsale me rétrécit quand j'entends un freak folklorique déclarer concernant la guitare électrique : «C'est pas acadien ». Je ne peux que répondre : «Je m’en goddam ben.» (Leblanc, Moncton Mantra: 104)

On y relève un désir d'éloignement de ce que Marc Augé identifie comme «l'individualité de synthèse » (Augé 1992 : 32), c'est-à-dire une identité vulgarisée, globalisée, qui se regroupe sous un article défini : l'Acadien. Moncton Mantra est un roman autobiographique qui relate la prise de conscience littéraire et sociale qui s'est effectuée à Moncton et en Acadie durant les années 1970, en plus de retracer le parcours personnel du protagoniste vers l'écriture. De l'état d'angoisse profonde à l'épiphanie, le personnage écrivain d'Alain Gautreau se sert de l'espace de la ville pour «étudie[r] [s]es maladies »(Leblanc, Comme un otage du quotidien: 11) et non pas les guérir, mais les mettre en mots, pour les rendre acceptables. ${ }^{10}$ Maladies identitaires, folkloriques, linguistiques, littéraires et spatiales, les maux sont nombreux, mais la cure est unique, et elle réside dans la définition même des maux, dans ce qui est considéré «normal» et ce qui l'est moins. Afin de guérir, le poète ne se redéfinira pas lui-même, mais s'appliquera à redéfinir les «possibles » de son identité acadienne, selon de nouveaux principes d'individualité.

C’est pourquoi la poésie de Leblanc, plus que tout autre texte acadien, aura cartographié Moncton comme ville acadienne, afin de la montrer sous un nouveau jour que celui de l'unilinguisme anglais, et de placer l'Acadie dans un espace autre que l'espace traditionnellement rural et folklorique, mais aussi, avant tout, de placer le je au cœur de l'espace, et non plus le nous :

Rue Dufferin. Je répète le nom de ma nouvelle rue, rue Dufferin, que je commence à aimer follement, que je commence à habiter pour de vrai dans mon corps, que j'habite dans le bruit des voisins, dans les grands arbres devant la maison, que j'habite dans le trafic de ma rue, que j'habite dans les paroles de Roland, que j'habite dans mon bain chaud, dans les musiques de mon stéréo, dans les visites magiques d'Anne-Marie, que j'habite dans l'idée que j'en ai et que je m'en fais. (Leblanc, Moncton Mantra: 84)

Les textes de Leblanc rendent l'espace urbain habitable en Acadie, nouvelle forme d'habitation dans l'espace qui devient possible par l'idée «d'en faire ce qu'on veut bien » (Leblanc, Éloge du Chiac: 40) :

\footnotetext{
${ }^{10}$ Raoul Boudreau explique : «Gérald Leblanc est très conscient de l'image négative de Moncton et de son déficit de reconnaissance, mais aussi des immenses possibilités de ce désert, de cette distance, de cette anarchie de l'extrême frontière. Dans cette tentative de faire de Moncton un lieu habitable, le poète commence avec un solde négatif, mais il va faire, selon le principe connu du retournement du stigmate, de cette négativité même la base de sa construction symbolique. » (Boudreau 2007 : 40).
} 


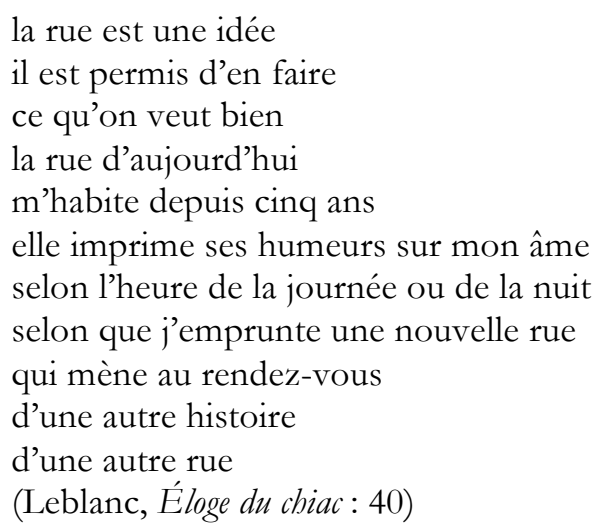

Il est ici question de perspective, d'empirisme, d'angle et d'individualité avant d'être/afin d'être une question de collectivité. De la chambre, à l'appartement, à la rue, à l'autre rue, au campus, au Kacho, au café chez Duane, à la Cave à Pape et au Centre Culturel Aberdeen, l'Acadien - et avec lui une nouvelle image de l'Acadie, s'établissent fermement dans l'espace urbain. Pour revenir sur les propos d'Olivier Mongin, il s'agit de faire état d'un corps collectif formé d'une «infinité [de] parcours» (Mongin 2005 : 39-40). L'individualité martelée du personnage dans sa quête identitaire renferme une fonction exemplaire :

Je saute dans un taxi pour me rendre au Kacho. Sur place, je l'aperçois au bar. Il me fait signe d'approcher. Je lui fais part de l'immense party de la rue St. George et l'invite à m'accompagner. On passe chez moi avant pour prendre un peu de mescaline et écouter de la musique. L'univers vibre. Il me demande si je lui ferais un massage. Je lui masse le dos, les épaules. Soudain nous glissons sur le plancher, submergés par le désir.

Nous nous réveillons vers midi et nous vidons ce qui reste de mescaline dans le café. Au milieu de cette journée ensoleillée, une sirène de pompier éclate tout près. Nous sortons en sautant comme des enfants dans la rue Dufferin pour se rendre sur la rue St. George, entre Highfield et Weldon. Arrivés en face du 320, nous apercevons Lorenzo enveloppé dans un drap blanc. Il nous invite à déjeuner sur le toit. [...]

Après avoir quitté Lorenzo, nous continuons notre randonnée dans le quartier, excités et débordants comme des fous. Dans le stationnement de l'épicerie au coin de la Highfield et de la St. George, Roland m'arrête et me regarde droit dans les yeux.

— Je n'ai jamais été si heureux d'être en vie! (Leblanc, Moncton Mantra: 91-92)

Chez Leblanc, les protagonistes ne se rendent pas simplement dans un bar, ne tournent pas un simple coin de rue, ne s'arrêtent pas dans le stationnement d'une épicerie; ils vont au Kacho, tournent sur la St. George, arrivent à l'épicerie au coin de la Highfield et de la St.George.

Dans le roman Moncton Mantra, la ville est un personnage à part entière, et elle est aussi le sujet principal de la poésie. Elle est une question posée dans le texte tout en étant implicitement la réponse :

qu'est-ce que ça veut dire, venir de Moncton ? une langue bigarrée à rythmique chiac, encore trop proche du feu. la brûlure linguistique. Moncton est une prière américaine, un long cri de coyote dans le désert de cette fin de siècle. Moncton est un mot avant d'être un lieu ou vice versa dans la nuit des choses inqiuétantes. Moncton multipiste : on peut répondre fuck ouère 
off et ça change le rythme encore une fois. qu'est-ce que ça veut dire, venir de nulle part? (Leblanc, l'Extrême frontière : 161)

La poésie de Gérald Leblanc, par sa cartographie systématique des lieux dans le but de rendre la ville « habitable », nous rappelle les propos d'Olivier Mongin :

Si la ville est cette unité symbolique qui rappelle une mémoire et qui anticipe un avenir, elle exige simultanément des lieux-seuils, des entre-deux permettant à des discontinuités de prendre forme. "La ville" serait d'abord ceci : l'instauration commune d'une référence. (Mongin $2005: 11$ )

C'est par la cartographie de ce nouvel espace acadien que les textes de Leblanc transposent l’individualité dans le collectif. Chez Leblanc, le corps qui se déplace dans la ville, nommant ses lieux, y insérant sa langue, ses goûts, ses amours, son rythme, montre que cette existence est possible dans sa forme collective - du moment que chacun y trouve son rythme.

ma ville est une ville de mots mon cœur est un cœur de quête mon corps est une expérience physique mes mots que sont ma vie et mon cœur et mon corps (Leblanc, Je n'en connais pas la fin: 58)

Dans l'espace urbain, l'identité acadienne du poète se trouve face à une multiplicité de possibles. Elle est moins sujette à un consensus collectif, mais plus ouverte à la pluralité du je ou à l'individualité : «ma ville », «mon cœur», «mon corps », « mes mots ». Plutôt que par un nous, l'identité s'exprime désormais par un je, et au sein de l'espace monctonien, l'identité acadienne collective se voit attribuée les caractéristiques du kaléidoscope. Les mots ne sont plus simplement au service du collectif, et les repères traditionnels éclatent. Les textes de Leblanc invitent à prendre « conscience du rythme » (Leblanc, Je n'en connais pas la fin: 72) individuel, de la pensée, de son corps, et des couleurs de l'espace. Dans le dernier recueil publié de son vivant - aboutissement obligé du parcours littéraire, les mots et l'image kaléidoscopique renvoyée par l'espace ont atteint la symbiose :

la lumière nous inspire
à calculer son effet
nos échanges mathématiques
les codes sonores
déchiffrement ludique
l'âme du bleu la lune
nos rapports à son cycle
son rythme et nous
(Leblanc, Techgnose: 87 )

Chez Leblanc, «l'univers vibre»(Leblanc, Moncton Mantra: 91) «l'espace [...] pulse » (Leblanc, Techgnose: 83), tout est rythme individuellement intériorisé, subissant un traitement empirique personnalisé dans le quotidien de l'existence. Dans l'urbain, l'être est entièrement sensoriel. 
sous la pluie la ville sous ma peau

mon quartier glisse vers le bleu trempe

je pensais à toi quand j'entendais Stereolab

la permission d'énergie

le feu la poésie le mix

l'échange d'idées de toi là-dedans

je pensais aux combinaisons au loop

sous la pluie

(Leblanc, Techgnose : 54)

Inspiré par les possibilités infinies de l'espace urbain, le poète choisit de ne plus faire de l'identité collective un fardeau mais une expérience. Sans vouloir baliser l'identité, le poète se sert plutôt de la ville pour définir de nouveaux repères identitaires, incarnés dans des «lieux-seuils » (Mongin 2005 : 11), ou encore, selon les mots d'Herménégilde Chiasson, des « îlots littéraires » (Leblanc, L'Extrême Frontière: 9) :

Multidimensionnelle, l'expérience urbaine doit [...] être déclinée à plusieurs niveaux : celui du corps, celui de l'habiter, celui de la scène publique, celui de la vie politique, mais aussi celui de l'appartenance à la Terre dans un monde globalisé. La réflexion sur la condition urbaine va de pair avec la question du corps : corps physique, corps planétaire et corps urbain doivent faire tenir ensemble des «éléments » qui ne s'accordent pas naturellement, à commencer par les plus «élémentaires ». [...] Au milieu de la ville, nous y sommes toujours, car les exigences de l'expérience urbaine persistent: il nous faut habiter, vivre dans un monde «soutenable », créer une durée publique, inventer des scènes et des théâtres. (Mongin 2005 : 298)

$\mathrm{Au}$ cours des 25 années que couvrent les publications de Leblanc, ses textes auront permis l'instauration complète - amorcée par Arsenault - de l'urbain comme espace identitaire possible au sein de la littérature. Ils auront également introduit une nouvelle façon d'envisager le collectif dans le texte acadien, à savoir l'expérience individuelle d'une déambulation urbaine s'exprimant au quotidien. Ceci aura eu pour effet de libérer le texte, et d'initier un champ d'exploration plus large sur le plan des formes et des contenus. Ainsi, au fil des années, de nombreux écrivains auront, à leur façon, emboîté le pas à Gérald Leblanc (Boudreau 2007 : 48), ce qui fait que, comme l'affirme François Paré, « sur le plan littéraire, l’Acadie, c’est [...] surtout Moncton!» (Paré 2009 : 34).

\section{Références bibliographiques :}

Les cent lignes de notre américanité. Actes du colloque tenu à Moncton du 14 au 16 juin 1984, collectif. 1984. Moncton : Les Éditions Perce-Neige.

Arsenault, G. 1973. Acadie Rock. Moncton : Les Éditions d'Acadie.

Augé, M. 1992. Non-lieux. Introduction à une anthropologie de la surmodernité. Paris : Éditions du Seuil.

Bolduc, Y. « Poésie acadienne contemporaine ». Québec français. 60 (déc. 1985) : 52-53. 
Boudreau, R. 1998. «L'actualité de la littérature acadienne ». Tangence. 58 : 8-18.

Boudreau, R. 2007. "La création de Moncton comme "capitale culturelle" dans l'œuvre de Gérald Leblanc ». Revue de l'Université de Moncton. 38 (1) : 33-56.

Boudreau, R et M. White. 2009. "Gérald Leblanc : écrivain cartographe » dans Paysages imaginaires d'Acadie. Un atlas littéraire, sous la direction de M.-L. Lord et D. Bourque. Moncton : Institut d'études acadiennes / Chaire de recherche en études acadiennes.

Bruce, C. 2005. «Gérald Leblanc et l'univers micro-cosmopolite de Moncton». Études Canadiennes/Canadian Studies. $58: 205-220$.

De Certeau, M. 1990. L'Invention du quotidien. t. I. Arts de faire. Paris : Gallimard. [1980]

Doyon-Gosselin, B. 2007. «Le déplacement des références identitaires et des balises territoriales dans Variations en $B$ et $K$ de France Daigle » dans Balises et références. Acadies et francophonies, sous la direction de M. Pâquet et S. Savard. Sainte Foy : Les Presses de l’Université Laval : 371-393.

Giroux, M. 1992. "Sur l'écriture : rencontre avec deux poètes acadiens ». Studies in Canadian Literature/Études en littérature canadienne. 17 (2) : 148-165.

Leblanc, G. 1981. Comme un otage du quotidien. Moncton : Les Éditions Perce-Neige.

Leblanc, G. 1988. L'Extrême Frontière. Poèmes 1972-1988. Moncton : Les Éditions d'Acadie.

Leblanc, G. 1993. Complaintes du continent. Poèmes 1988-1992. Moncton [et] Trois-Rivières : Les Éditions Perce-Neige [et] Écrits des Forges.

LeBlanc, G. 1995. Éloge du chiac. Moncton : Les Éditions Perce-Neige.

LeBlanc, G. 1997. Moncton Mantra. Moncton : Les Éditions Perce-Neige.

Leblanc, G. 1999. Je n'en connais pas la fin. Moncton : Les Éditions Perce-Neige.

Leblanc, G. 2003. «L'alambic acadien : identité et création littéraire en milieu minoritaire », dans L'Acadie plurielle. Dynamiques identitaires collectives et développement au sein des réalités acadiennes, sous la direction d'A. Magord. Université de Moncton / Université de Poitiers : Centre d'études acadiennes : 517-522.

LeBlanc, G. 2004. Techgnose. Moncton : Les Éditions Perce-Neige.

Leblanc, R. G. 1972. Cri de terre. Moncton : Les Éditions d'Acadie.

Lord, M.-L.. 2006. «Identité et urbanité dans la littérature acadienne » dans Regards croisés sur l'bistoire et la littérature acadiennes, sous la dir. de S. Jaumain et M. Frédéric. Bruxelles : Peter Lang : 67-85.

Mongin, O. 2005. La Condition urbaine, Paris : Seuil.

Paré, F. 2001. Les Littératures de l'exiguité. Ottawa : Le Nordir. [1993]

Paré, F. 1998. «Acadie City ou l'invention de la ville ». Tangence. 58 : 19-34.

Paré, F. 2009. «La poésie acadienne contemporaine ». Nuit Blanche. 115 : 34-38.

Plantier, R. 1988. «L'identité acadienne et l'américanité », Cultures du Canada français, 5 : 129-145. 